\title{
Conservare e divulgare: la Biblioteca dell'Istituto storico di Modena dalle origini agli anni Ottanta
}

L'Istituto storico di Modena fin dalle origini nel 1950 considera compito essenziale la raccolta di documentazione sia archivistica sia libraria. La biblioteca nasce quindi e si sviluppa con acquisti e doni, tra problemi di spazio e di personale, difficoltà economiche, progressiva apertura a un pubblico ampio. E costante la consapevolezza di operare in un contesto plurimo: rapporti con gli altri Istituti storici a livello nazionale e soprattutto una forte relazione con la realtà bibliotecaria locale, alla ricerca di coerenze, specificità, visioni comuni.

Since its origins in 1950, the Istituto storico di Modena considers its main task collecting both books and documents. The library is born and grows with donations and purchases, needs more space and staff, has financial difficulties and is gradually opening to a wide audience. We are aware we are working in a complex context made of relations with other national historical institutes, and especially a strong relationship with the local libraries, in order to build coherence, specificity, common visions.

Una raccolta libraria accompagna fin dalle origini la vita dell'Istituto storico di Modena: una presenza inizialmente discreta, e negli anni sempre più evidente per la crescita del patrimonio e la partecipazione al sistema bibliotecario della città. Scorrendo i verbali del Consiglio direttivo e dell'Assemblea dei soci', già nel 1958 si riscontra la nomina di una Commissione specifica per la biblioteca, presieduta da Ennio Pacchioni allora presidente dell'Istituto stesso: segni inequivocabili di un interesse per la documentazione a stampa che si va affiancando a quella archi- 
vistica. È la realizzazione concreta di quanto già previsto dallo Statuto approvato nel 1950, che prevede all'articolo 2 come primo compito «la ricerca, raccolta, conservazione e classificazione della documentazione archivistica, giornalistica e libraria» relativa al movimento di liberazione'. Mentre l'archivio potrà contare su un'attenzione costante negli anni grazie alla presenza continuativa di una collaboratrice come Ilva Vaccari, e per vari anni di Filippo Valenti vice direttore e in seguito direttore dell'Archivio di Stato, la biblioteca vedrà un frequente avvicendarsi di responsabili, collaboratori, membri di commissione, insegnanti distaccati ${ }^{3}$; tuttavia si farà rapidamente strada la consapevolezza dell'importanza del patrimonio di libri e riviste, e del suo intreccio fruttuoso con la raccolta di documenti.

Questo percorso è favorito dal clima generale che vede in tutto il paese, tra gli anni Cinquanta e Sessanta, riprendere slancio l'interesse per la lettura e la biblioteca, dopo che l'esperienza delle biblioteche popolari di inizio Novecento aveva subito una seria battuta d'arresto nel ventennio fascista e nel periodo bellico [Montecchi 2012, 42-45]; anche in provincia di Modena gli enti locali iniziano a porsi il tema del servizio di

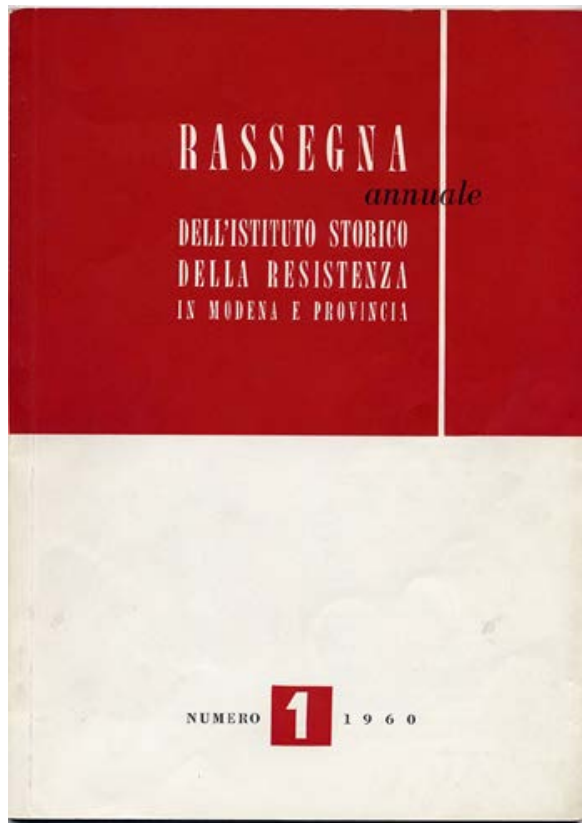

Rassegna annuale pubblica lettura in termini moderni, con obiettivi di istruzione e di svago, spesso su sollecitazione di associazioni di cittadini e gruppi spontanei [Romagnoli 2012, 91-101]. Si può quindi lasciare a loro il compito di percorrere questa strada, mentre l'Istituto concentra il suo impegno sulla salvaguardia della memoria e la divulgazione della conoscenza storica. 


\section{Le origini}

Nel 1959 all'Assemblea dei soci si parla di un patrimonio consistente in 120 titoli sulla Resistenza, oltre alla rassegna "Il movimento di liberazione in Italia"; si inizia anche a citare un bilancio specifico per gli acquisti, se pure di misura limitata 4 Non si può quindi ancora parlare di una vera biblioteca, e non desta meraviglia la mancata inclusione nella pubblicazione Le biblioteche dell'Emilia: storia patrimonio attività, curata dal nuovo Soprintendente bibliografico [Samek Ludovici 1959], dove invece compare una scheda relativa alla Biblioteca Ferrarini che più avanti entrerà in stretto rapporto con la biblioteca dell'Istituto ${ }^{5}$.

L'Assemblea dei soci del 1962 registra una articolata riflessione sulla biblioteca da parte del presidente, che nella sua relazione generale sottolinea con forza $\mathrm{i}$ problemi dell'aggiornamento patrimoniale e della sede. La biblioteca ha già un impianto solido:

Quello che importa ora è il suo continuo aggiornamento e la raccolta di opere che difficilmente si trovano sul mercato librario in quanto divenute rare ed introvabili: per questo bisognerà seguire l'esempio dell'Istituto nazionale che si vale a tal scopo del mercato antiquario del libro. Altra attività utile l'Istituto nostro potrà esplicarla nel consigliare le biblioteche comunali, popolari e locali indicando libri e pubblicazioni adatti a costituire una sezione apposita dedicata alla storia contemporanea italiana ed alla Resistenza. Questo stiamo già facendo con Sassuolo, dove per lodevole iniziativa di quella Amministrazione comunale si sta organizzando un'ottima biblioteca.

La questione del patrimonio si pone quindi non solo a livello interno, ma anche in rapporto alla crescita complessiva delle biblioteche del territorio: grazie a una "bibliografia della Resistenza" commissionata un paio d'anni prima e all'epoca completata, è possibile suggerire acquisti alle biblioteche comunali e scolastiche. L'aumento patrimoniale sia della biblioteca sia dell'archivio accentua il problema, già presente, della ristrettezza della sede, che il segretario Ilva Vaccari considera ostacolo da superare con urgenza:

4 Molti anni dopo, nel 1990 in occasione del $40^{\circ}$ anniversario dell'Istituto, Pietro Alberghi parlerà di 500 libri a fine 1961, 1.000 a fine anni Sessanta, 2.110 a fine 1971, 4.475 a fine 1980, 6.485 a fine 1986, 7.000 nel 1990. P. Alberghi, I quarant'anni di vita dell'Istituto, "Rassegna di storia dell'Istituto storico della Resistenza e di storia contemporanea in Modena e provincia" (d'ora in poi "Rassegna di storia"), nuova serie, 9, 1990, 82-83.

5 Secondo l'estensore della scheda, Armando Pellati, «la biblioteca, che ha soprattutto carattere popolare, è costituita da più di 6.000 volumi, in prevalenza di genere narrativo e divulgativo; non mancano opere di cultura e collezioni di classici di discreto valore, ma quasi nullo è attualmente l'incremento annuo, per l'insufficienza dei mezzi. Esistono due cataloghi aggiornati, uno per autori e l'altro per materie». Da Metella Montanari [Montanari 2012] apprendiamo che Pellati era all'epoca (1959) commissario governativo, dato che fino a quell'anno l'Istituto Ferrarini era stato affidato alla gestione del Proweditorato agli studi. 
Se l'Istituto deve mirare a divenire anche sede di consultazione per gli studi sulla Resistenza, e vuole farlo con orari nuovi, agevoli a tutti, anche a coloro che lavorano (cosa ora preclusa negli archivi di stato e comunali), cioè con larga e prolungata apertura al pubblico, che così incoraggiato ed agevolato, si spera affluirà numeroso, ci deve essere una sede larga, comoda e decorosa, degna insomma della Resistenza.

È presente il sindaco Corassori che promette una sede più idonea, mentre il consigliere Lucchi avanza una proposta ardita, che non troverà seguito negli anni a venire: collocare l'Istituto nel Palazzo dei musei, trasferendo Galleria e Pinacoteca estensi in un nuovo edificio statale in un viale intitolato agli Este.

\section{La "biblioteca di storia contemporanea"}

Nel luglio dello stesso anno, il Consiglio direttivo ha notizia che il Comune ha revocato un finanziamento destinato alla Biblioteca Ferrarini, e ha invece intenzione di incaricare l'Istituto di allargare la propria biblioteca e darle il preciso carattere di "biblioteca di storia contemporanea". Per la prima volta le carte mostrano l'avvio di un ciclo che si completerà vent'anni dopo: il glorioso Istituto Ferrarini ha ormai esaurito la sua funzione, e l'Istituto storico prende in carico il ruolo di riferimento per la documentazione e lo studio della storia contemporanea, così come le biblioteche pubbliche assumono il compito di un servizio di base alla collettività ${ }^{6}$. A fronte della soddisfazione per il significato di questo pronunciamento dell'istituzione, ci sono ovviamente le preoccupazioni: la Vaccari sottolinea la portata di un tale impegno e la conseguente necessità di un'organizzazione diversa:

La nuova sede non può avere meno di cinque ambienti poiché la biblioteca sola dovrà contare, separatamente dall'Istituto, almeno di una vasta e tranquilla sala di consultazione e lettura, una saletta di prestito e un piccolo ufficio per le operazioni di immatricolazione, registrazione, collocazione.

Sulla biblioteca di storia contemporanea pare sussista pure un interesse dell'Università degli studi, che vorrebbe ripristinare la laurea in Scienze politiche. La nuova sede ci sarà dall'8 settembre 1963, in via Falloppia 45. La biblioteca è definita "istituenda", "progettata": c'è quindi consapevolezza dell'avvio di 


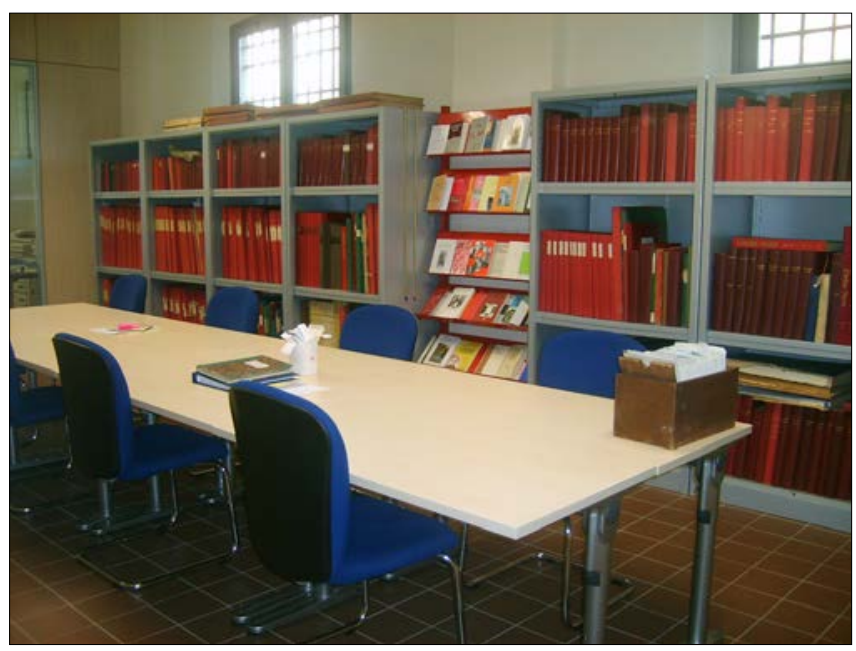

La sala di studio oggi

una nuova fase, e della necessità di avere aiuto dalle istituzioni: il presidente Pacchioni chiede supporto a Comune e Provincia, scrive al sindaco Rubes Triva per avere uno schema di convenzione e all'Ispettore generale bibliografico del Ministero della Pubblica Istruzione, Guido Stendardo, per chiedere consiglio riguardo al progetto di associarsi alla Federazione delle Biblioteche popolari. Con quest'ultimo, prende l'occasione per ringraziare dell'invio da parte del Ministero di oltre una settantina di volumi «residuati fascisti»»: notazione interessante, perché ulteriore riconoscimento del ruolo dell'Istituto nell'ambito della conservazione della documentazione storica e in particolare del materiale d'epoca fascista, che evidentemente esce dagli scaffali "d'uso" dove ormai rappresenta una presenza imbarazzante, per cercare una sede dove possano esercitarsi ricerca e analisi critica.

Altro raccordo considerato importante è quello con le biblioteche affini: nel Direttivo dell'ottobre 1964 alcuni consiglieri riferiscono sugli esiti dell'Assemblea generale dell'Istituto nazionale cui hanno partecipato, affermando tra l'altro:

Il senatore Parri [Presidente] ha anche ravvisato quale premessa e condizione per un lavoro organico da parte degli istituti locali, un metodo uniforme di archiviazione del materiale documentario e, in materia di biblioteche specializzate nella Resistenza, comunicazioni reciproche per quanto riguarda le consistenze attuali e gli acquisti futuri.

Come modello, "biblioteca pilota", viene citata la Biblioteca Einaudi di Dogliani: la stessa che prenderanno a riferimento, fra i tanti, le biblioteche di quartiere di Modena di cui si sta progettando l'apertura. In effetti la Biblioteca di Dogliani, 
inaugurata da ben poco tempo - il 29 settembre 1963 - rappresenta il nuovo tipo di biblioteca "concepito come un centro di cultura vivo e aperto, bene inserito nella comunità, moderno e capace di parlare immediatamente alla gente di oggi», che, non potendo contenere tutto, deve però contenere almeno "il seme di tutto". Può essere quindi un riferimento solo ideale per la biblioteca specializzata: Delio Cantimori, impegnato a stendere l'introduzione alla Guida alla formazione di una biblioteca che discende dall'esperienza di Dogliani, parla della conoscenza storica come «necessaria per chi vuol essere cittadino e uomo consapevole e attivo, come la conoscenza delle matematiche è necessaria a chi vuol costruire un ponte o scavare una galleria; e ad un certo momento deve essere specializzata» [Guida alla formazione 1969, XXV-XXVII e 531-658]. In questo punto si individua il possibile riferimento per la biblioteca dell'Istituto.

Il problema dei finanziamenti percorre tutte le riunioni di questi anni, tra la difficile definizione di una cifra sul bilancio interno, l'attesa di contributi da parte del Comune che tardano ad arrivare (si cita lo stanziamento di un milione di lire a dicembre 1965), il progetto di acquisto di libri da parte del Comune per farne un deposito presso l'Istituto. Ugualmente pressante è il tema del personale «fisso e qualificato»: in corrispondenza dei finanziamenti, è urgente definire un piano di acquisti e progettare schedatura e riordino, mentre le attribuzioni interne di responsabilità ai consiglieri portano spesso a scarsa disponibilità di tempo e successive rinunce.

All'Assemblea dei soci del febbraio 1965 è presente e interviene Ermanno Gorrieri, che riserva una parte del suo discorso a biblioteca e archivio:

Sottolinea le due principali funzioni dell'Istituto: di divulgazione e, soprattutto, di ricerca e raccolta delle fonti. Occorre intensificare la seconda, che sino ad ora è stata cura particolare quasi esclusivamente della signorina Ilva Vaccari; a questi studi sarebbe bene interessare i giovani attirandoli con borse di studio o iniziative analoghe.

Attraverso i verbali del Direttivo è possibile ripercorrere alcune tappe dello sviluppo patrimoniale di quegli anni: nel 1967 la casa editrice Einaudi ha donato 69 volumi', nel 1968 il Comune di Modena affronta la spesa di L. 1.583 .700 per l'intero catalogo Feltrinelli Reprint da depositare in Istituto (una settantina di titoli ${ }^{10}$ ); nel 1969 arrivano in dono annate della "Gazzetta dell'Emilia" e «giornaletti del periodo repubblichino»; successivamente, si decide l'acquisto degli Annali Fel- 


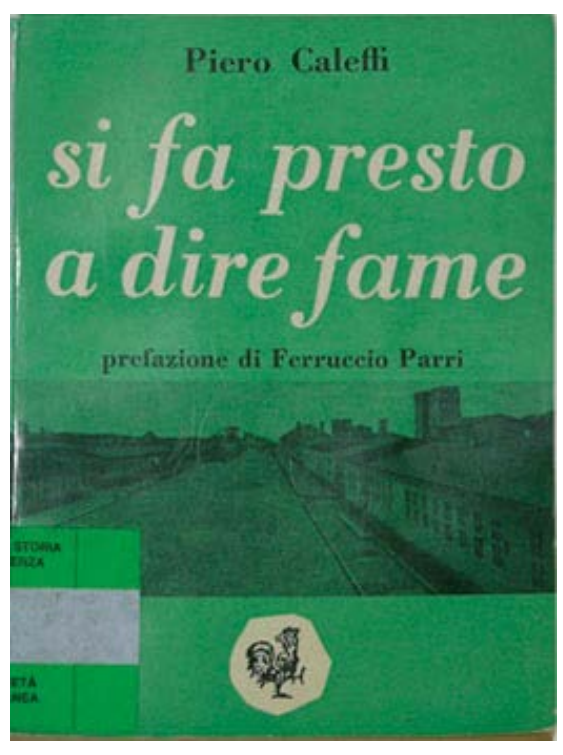

trinelli e di repertori di lavoro come il $\mathrm{Ca}$ talogo dei libri in commercio; Pacchioni nel 1972 scrive a Tristano Codignola de La Nuova Italia chiedendo aiuto per acquistare annate arretrate del periodico "Il Ponte" anche a prezzi di antiquariato purché ragionevoli". Interessante è l'acquisizione di libri editi durante il regime fascista di proprietà del Liceo Muratori, e qui valgano le considerazioni fatte sopra per i volumi ceduti dal Ministero dell'Istruzione: i libri d'uso scolastico durante il ventennio stanno lentamente trovando una sistemazione dove sia evidente il loro status di documenti per la ricerca storica.

Evidentemente è considerato importante

l'arrivo di libri e documenti, benché questo aggravi l'arretrato di documenti da catalogare. Nel frattempo, nel 1969, si è deciso il trasferimento della biblioteca nella sala delle riunioni perché «meglio riscaldata e più agevole per i giovani che la frequentano»; ci si pone inoltre per la prima volta il tema della redazione di un Regolamento per la biblioteca e uno per l'archivio.

All'esterno la situazione sta mutando, anche ad opera del Comune che negli anni Sessanta ha percorso due strade importanti e parallele per allargare la ristretta platea di lettori: otto edizioni del festival del libro economico tra 1962 e 1969 hanno richiamato l'attenzione sul libro come veicolo culturale a basso costo [Bellei 2012], e dalla seconda metà del decennio sono aperte le prime biblioteche di quartiere, compresa la Civica di corso Canalgrande ${ }^{12}$. Occorre quindi tener conto del contesto mutato e porsi il problema di un nuovo possibile rapporto con questi servizi; a febbraio 1971 un consigliere cita due temi al riguardo: innanzitutto, l'importanza delle biblioteche comunali e la conseguente opportunità di essere presenti nel loro patrimonio con le pubblicazioni dell'Istituto; in secondo luogo, l'esistenza di commissioni per le biblioteche comunali di cui potrebbero far parte anche membri dell'Istituto. Si tratta ormai, da tutte e due le parti, di istituti strutturati per i quali si analizzano le possibilità di raccordo. 


\section{Organizzazione interna del patrimonio librario e apertura al pubblico}

Anche i temi strettamente professionali hanno un riflesso nei documenti degli organi collegiali. Ci si pone il problema della schedatura sia delle riviste, sia delle tesi di laurea considerate parte essenziale del patrimonio: per queste ultime Pacchioni scrive nel 1972 all'Istituto romano per la storia d'Italia dal fascismo alla Resistenza per chiedere informazioni sui criteri usati. Nello stesso anno Francesca Tosi, responsabile della biblioteca dell'Istituto nazionale per la storia del movimento di liberazione in Italia, invia copia del soggettario specifico per gli istituti, ai fini di un catalogo unico nazionale. Si tratta di uno schema decimale, ancora in bozza, che prevede:

- $\quad$ 1. Storia tra le due guerre mondiali,

- 2. La seconda guerra mondiale (il più sviluppato),

- 3. Dopoguerra (conciso),

- $\quad 0$. Storia anteriormente alla prima guerra mondiale (molto conciso).

La Commissione biblioteca dell'Istituto avanza obiezioni sul soggettario per la sua debolezza relativamente al periodo precedente il fascismo e la Resistenza, considerato invece fondamentale: «La Resistenza italiana infatti inizia fin dall'indomani del 28 ottobre 1922 e non già dall' 8 settembre 1943 quando comincia la fase della lotta armata». Si decide quindi di utilizzare il soggettario nazionale integrandolo con quello preparato da Rosanna Giugni, insegnante distaccata. Al di là dell'aspetto tecnico, la discussione è interessante perché evidenzia, da un lato, la consapevolezza di dover inquadrare il fenomeno resistenziale in un contesto più ampio di premesse e conseguenze; dall'altro, una concezione dell'antifascismo storico come "semplice" prima manifestazione della futura Resistenza, vista con esso in totale continuità: concezione che durerà a lungo e sarà meglio inquadrata in ricerche successive [Rapone 2012].

I primi anni Settanta, nei quali si sta consolidando e ampliando la rete delle biblioteche di pubblica lettura, sono determinanti anche per l'affermazione della biblioteca dell'Istituto. Non è un caso se si esaminano problemi tecnici prima assenti dalle riflessioni: si sta infatti progettando l'apertura ufficiale al pubblico nella sede di via Falloppia, che interverrà 1'11 aprile $1973^{13}$; il Regolamento prevede l'apertura nel pomeriggio dei giorni dispari dalle ore 15 alle ore 18, la sola 
consultazione in sede, l'ammissione per gli studenti degli istituti superiori e universitari e gli studiosi in genere maggiori di 16 anni. È da credere che la biblioteca abbia subito riscontrato una buona frequenza, se già a metà 1974 l'orario è ampliato a tutti i pomeriggi dei giorni feriali e il prestito è introdotto, anche se «per i soli casi di effettiva necessità di ricerca». Una persona è incaricata dell'assistenza al pubblico, mentre si contatta l'Archivio di stato per l'organizzazione di "corsi per apprendisti archivisti e bibliotecari". L'Istituto decide anche di associarsi alla Federazione italiana della biblioteche popolari, che fornisce supporto ai soci fino al 1974 per l'acquisto diretto di libri, e in seguito per «il servizio di consulenza per l'acquisto libri, la pubblicazione

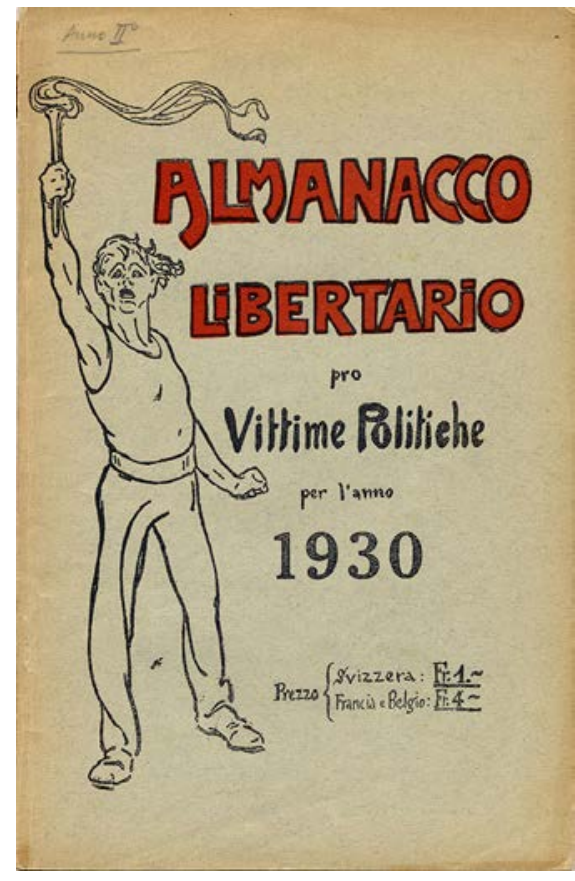

Almanacco libertario pro vittime politiche, 1930 dell'Annuario bibliografico, la pubblicazione del manuale del bibliotecario, i corsi residenziali per bibliotecari presso il Centro residenziale, la pubblicazione della rivista "La cultura popolare", l'adozione di nuovi schedari e la standardizzazione relativa, il servizio di consulenza per l'impianto di nuove biblioteche e per la sistemazione delle vecchie, l'offerta di pacchi dono, ecc.».

A conclusione della sua attività, la Giugni presenta una relazione dettagliata sul lavoro svolto per la biblioteca ${ }^{14}$, in cui oltre a dar conto dell'attività del periodo fornisce una fotografia della biblioteca in quel momento. I libri occupano gli scaffali da A a F (parte) con 6 ripiani ognuno; le riviste, gli scaffali da F (parte) a G, più gli "scaffali in ferro" a e b, per "Gazzetta dell'Emilia" e Reprint Feltrinelli. I lavori ultimati comprendono: registro d'ingresso, divisione dei libri per materie, catalogo per autori, catalogo d'ingresso [sic], soggettario per alcune materie, schedoni per le riviste, riordino in contenitori e schedatura per gli opuscoli, avvio del lavoro sui doppi, inizio della compilazione delle schede per gli autori successivi al primo e per i curatori. 


\section{Perfezionamento dello sviluppo patrimoniale e valorizzazio- ne}

A fine anni Settanta, mentre è presidente dell'Istituto don Nino Monari, la responsabilità della biblioteca è in capo al consigliere Pietro Alberghi, che tra l'altro propone di coinvolgere nelle proposte d'acquisto esperti e studiosi, di selezionare meglio gli acquisti in base alle caratteristiche specifiche dell'Istituto, di rivolgersi alle librerie più convenienti sul piano economico; l'attività si avvale della insegnante distaccata Paola Nava, successivamente sostituita dallo stesso Alberghi. Nel Direttivo di inizio 1980, un allegato al verbale a firma Vaccari sintetizza il programma per i vari settori dell'Istituto:

Biblioteca: è fondamentale un progetto di acquisto, che punti su testi specializzati sul periodo considerato, su ricerche locali fatte in tutta Italia ma anche in Europa, e su testi che affrontino il dibattito metodologico sulla ricerca storica e sul rapporto con l'insegnamento della storia. È indispensabile poi pubblicizzare sia l'archivio che la biblioteca; per questo, oltre ai soliti appelli alle radio-televisioni locali e alle scuole, si potrebbe pensare: 1) di fare qualche iniziativa di lancio di libri acquistati, ovviamente i più noti e i più ricchi nel contenuto. 2) di andare direttamente nelle scuole, collegandosi con gli insegnanti delle classi dove si studia il fascismo, la resistenza, il dopoguerra e iniziare un prestito di libri dell'Istituto. Analoghe iniziative si possono fare nei quartieri o alle 150 ore per i lavoratori.

La Biblioteca ha evidentemente raggiunto un buon livello quanto a sede, patrimonio, strumenti e servizi; si tratta quindi da un lato di perfezionare lo sviluppo patrimoniale, dall'altro di valorizzarla il più possibile, soprattutto in direzione dei giovani.

Nel 1981 il patrimonio assomma a 4.820 volumi $^{15}$. È in questo momento che arriva la proposta dall'Istituto Ferrarini di cedere la propria biblioteca di libri e riviste in deposito perpetuo, dietro corresponsione della cifra di un milione di lire. Si decide per un sopralluogo e in seguito a questo, il 25 giugno, per l'acquisto con un esborso di L. 800.000. Una lettera del pre-

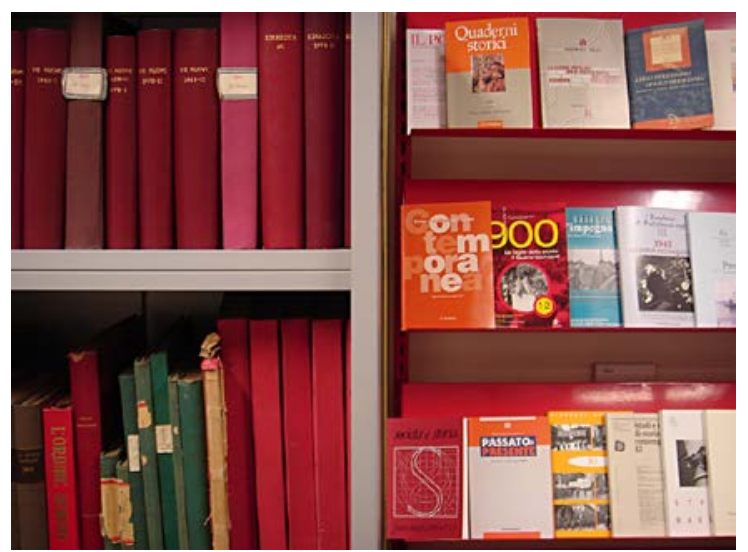

Periodici a disposizione in sala studio

15 Ismo, Arch. ist., Verbali degli organi collegiali 1981-1993, b. 2. 
sidente del Ferrarini ${ }^{16}$, in data 6 ottobre, fissa la cessione che comprende «testi sul fascismo e sull'antifascismo ed annate complete della Illustrazione italiana dal 1911 al 1942 e due annate della Rivista illustrata del popolo d'Italia». Indica inoltre le motivazioni che hanno indotto alla rinuncia:

Come le sarà noto la biblioteca Ferrarini, la cui costituzione risale ai primi anni del secolo, ha svolto una preziosa ed allora insostituibile funzione di diffusione della cultura, specialmente tra le classi sociali meno abbienti. Ora tale funzione è largamente assolta da altre istituzioni pubbliche e pertanto l'Istituto Ferrarini, al fine di non lasciare inutilizzato il proprio patrimonio librario, ha ritenuto di cedere all'Istituto storico della Resistenza modenese quei volumi che possono interessare gli studiosi che frequentano la vostra biblioteca.

La lettera precisa che si tratta di una parte della biblioteca del Ferrarini, e rimanda a un elenco allegato che purtroppo finora non è stato rintracciato; ugualmente, non sono state finora ricostruite le vicende che hanno consegnato il restante patrimonio all'attuale Laboratorio di poesia, cui afferisce pure l'Archivio dell'Istituto Ferrarini: si registra quindi oggi una divisione poco comprensibile di un patrimonio cresciuto unitariamente.

In assenza dell'elenco, si può considerare come fonte la descrizione effettuata sui registri d'ingresso dell'Istituto in due momenti: tra marzo e aprile 1982, con numeri dal 4370 al 4449, e tra aprile e luglio, con numeri dal 4480 al 4652: entrambe le serie riportano alla colonna provenienza: "Biblioteca Ferrarini", e inoltre i volumi fisici conservano etichette e timbri che ne denunciano il possesso originario. La lettera di cessione chiede esplicitamente che venga istituito presso l'Istituto un fondo dedicato, «in modo da ricordare una gloriosa e meritoria istituzione che ancora vive nel ricordo di molti modenesi»: il Fondo Ferrarini è in effetti costituito presso l'Istituto, e oltre ai 253 volumi sopra citati comprende altri numerosi volumi recanti numeri d'inventario sia più bassi sia più alti, con registrazione in ingresso che spazia dagli inizi degli anni Settanta fino ai primi anni Novanta; alcuni libri recano segni di provenienza varia ${ }^{17}$. Di questi altri arrivi non si è trovata per ora traccia documentaria.

All'Assemblea generale dei soci del dicembre 1981 il Presidente relaziona tra l'altro su questa acquisizione che comprende anche varie annate, se pure non tutte, della "Illustrazione italiana" tra il 1911 e il 1944, «offerte anch'esse dalla

\footnotetext{
16 Ismo, Arch. ist., Atti e corrispondenza generale 1981, b. 9.

17 Stefano Magagnoli ammette la mancanza di fonti certe per l'attribuzione di molti volumi al fondo, anche per la presenza di timbri spuri (es.: Istituto magistrale Regina Elena); nell'attesa che emerga una eventuale diversa documentazione, propone di mantenere l'unitarietà del Fondo, in nome della sua coerenza interna e della sua presenza ormai storicizzata [Magagnoli 1998]. Per l'inventario e l'analisi del Fondo Ferrarini si rimanda a questa pubblicazione.
} 
Biblioteca Ferrarini per una cifra poco più che simbolica» ${ }^{18}$.

Ormai la biblioteca è pienamente inserita nel sistema bibliotecario locale: alle riunioni della Commissione partecipano il direttore della Estense Ernesto Milano, per il Comune Maurizio Franciosi e Mauro Francia, per la Libreria Rinascita Giorgio Bettelli. Sul piano tecnico si solleva il tema di una catalogazione automatizzata; quanto alla missione specifica, si preme l'acceleratore sull'attività didattica nella convinzione che

la raccolta di volumi, documenti, fotografie, giornali avrebbe poco senso se non servisse a far conoscere ai giovani nati nel secondo dopoguerra gli anni turbinosi che noi più anziani abbiamo vissuto e, insieme alla loro conoscenza, non contribuisse ad instillare in essi il culto delle libertà democratiche, il senso della vita come impegno quotidiano nella difesa dei valori fondamentali dell'individuo e delle istituzioni conquistate a prezzo di sacrifici inenarrabili.

Nel 1981 l'Istituto riprende le pubblicazioni del periodico "Rassegna di storia dell'Istituto storico della Resistenza e di storia contemporanea in Modena e provincia" dopo una sospensione di ben dodici anni. L'occasione è propizia per fare il punto sulle diverse aree di attività e quindi dare notizie anche sulla biblioteca; si tratta in realtà di una interessante descrizione delle caratteristiche qualitative della biblioteca a quella data:

L'Istituto storico della Resistenza modenese è dotato, oltre che di un ricco archivio, di un'ampia ed aggiornata biblioteca (5000 volumi), specializzata in pubblicazioni di storia locale riguardanti il periodo fascista e la Resistenza. Accanto a queste, testi di consultazione generale, di storia d'Italia ed internazionale, attinenti al periodo dal primo ' 900 al secondo dopoguerra, volumi "in prestito" di storia nazionale e mondiale, i cui contenuti sempre abbiano a che fare con l'approfondimento dello studio del nostro secolo. In particolare, si è operata una divisione in sezioni, per facilitare la consultazione degli studiosi, in opere di storia generale, mondiale, europea, italiana (le più preziose e le enciclopedie non sono però comprese nel prestito, ma solo consultabili all'Istituto); in testi di storia del periodo fascista, dell'antifascismo italiano e straniero, della Resistenza italiana ed europea; in volumi sulla Germania dell'epoca nazista, sui lager, sugli ebrei; in libri sulle guerre mondiali e sull'Esercito italiano Esistono poi sezioni specifiche sui movimenti e partiti politici italiani, sul movimento operaio, sulla Chiesa cattolica; inoltre, da poco si è costituita una parte che mira ad approfondire la metodologia della ricerca, storica e sociale, che si va arricchendo sempre più di contributi teorici e ricerche sul campo. Della biblioteca fanno parte anche i periodici, tra $i$ quali spiccano quelli modenesi (la Gazzetta di Modena è la raccolta più esaurien-

18 L'attuale emeroteca dell'Istituto comprende sia questo periodico, sia la "Rivista illustrata del popolo d'Italia", citata nella lettera del presidente del Ferrarini. 
te), ancora una volta attinenti al periodo storico fascismo e Resistenza; ma non manca poi tutta una serie di periodici politici a carattere nazionale, insieme con le Riviste più significative (attuali) di storia nazionale elocale. Infine, ampio spazio è dato alle riviste pubblicate dagli Istituti storici della Resistenza, che esistono in altre città, e che compiono un'intensa opera politico/culturale sul piano della ricerca e della didattica ${ }^{19}$.

Sul piano quantitativo, invece, occorre fare riferimento alla ricerca sulle biblioteche modenesi effettuata nel 1982 per iniziativa dell'Assessorato alla cultura comunale, cui l'istituto aderisce compilando l'apposito questionario. Da questo documento, che si assume come finale per l'attuale ricerca, emerge che la biblioteca ha una previsione di spesa nell'anno di L. 5.000.000, occupa due dipendenti a tempo parziale, conta 5.171 volumi di cui 4.078 nella classe Dewey 900 (Storia) e 228 periodici, dispone di 182 metri di scaffalature e 10 posti lettura. La caratteristica

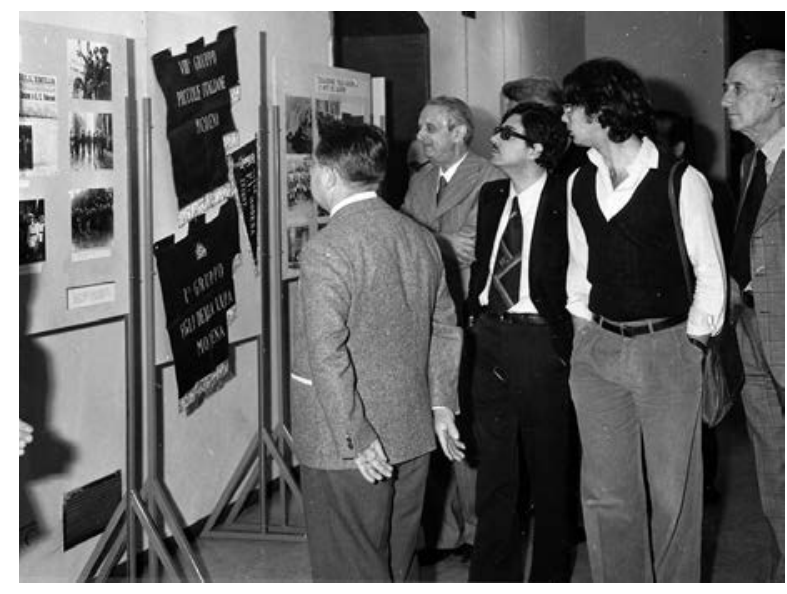

Mostra per il $30^{\circ}$ anniversario dell'Istituto, 1980 specifica è «biblioteca di storia contemporanea, con particolare riferimento alla storia locale e regionale» ${ }^{20}$. Tra difficoltà di vario tipo, la biblioteca continua il suo percorso e sempre più si specializza nel contesto ormai complesso degli istituti culturali della città.

19 Qualche notizia sulla biblioteca, "Rassegna di storia", nuova serie, 0, 1981,133.

20 Gli esiti dell'indagine si possono consultare in Assessorato alla cultura del Comune di Modena 1983, e in Biblioteche a Modena 1984, in particolare alle pp. 59-63: intervento di Pietro Alberghi, responsabile della biblioteca dell'Istituto storico della Resistenza. 


\section{Bibliografia}

Bellei M. 2012, “Cosa si legge e come si legge” a Modena negli anni Sessanta, in Montecchi G. e Manelli R. (a cura di) 2012.

Biblioteche a Modena. Stato attuale e ipotesi di sviluppo. Atti della giornata di studio, sabato 21 maggio '83 1984, Modena: Cooptip.

Guida alla formazione di una biblioteca pubblica e privata 1969, Torino: Einaudi

Assessorato alla cultura del Comune di Modena 1983, Indagine sulle biblioteche modenesi, Modena: Comune di Modena

Magagnoli S. 1998, Una biblioteca per il popolo. Il Fondo librario dell'Istituto di cultura popolare "Lodovico Ferrarini" di Modena, Modena: Istituto storico

Montanari M. 2012, Modena nel primo Novecento: l'Istituto Lodovico Ferrarini, in Montecchi G. e Manelli R. (a cura di) 2012.

Montecchi G. e Manelli R. (a cura di) 2012, Biblioteche e lettura a Modena e provincia dall'Unità d'Italia ad oggi, Bologna: Editrice Compositori.

Montecchi G. 2012, Leggere a Modena e in provincia dopo l'Unità d'Italia: la prima fioritura delle biblioteche popolari, in Montecchi G. e Manelli R. (a cura di) 2012.

Rapone L. 2012, Saggio introduttivo, in A. Osti Guerrazzi, G. Taurasi, P. Trionfini (a cura di) 2012, Dizionario storico dell'antifascismo modenese, Milano: Unicopli

Romagnoli P. 2012, Fonti per lo studio delle biblioteche del territorio modenese tra Otto e Novecento: le carte dell'Archivio della Provincia di Modena, in Montecchi G. e Manelli R. (a cura di) 2012.

Samek Ludovici S. 1959, Le biblioteche dell'Emilia: storia patrimonio attività, Modena: Artioli.

\section{Risorse}

Istituto storico di Modena www.istitutostorico.com

Polo bibliotecario SBN modenese www.bibliomo.it 